DO-TH-94/06

March 1994

\title{
The Fermion Determinant in External Scalar Fields: Exact Results and Large Mass Approximation
}

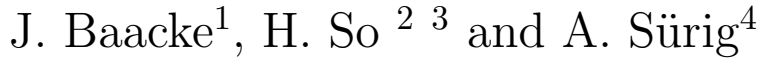 \\ Institut für Physik, Universität Dortmund \\ D - 44221 Dortmund, Germany
}

\begin{abstract}
We present results for the fermion determinant in an external Yukawa-coupled scalar field, a quantity relevant to instanton and sphaleron transition rates as well as bubble nucleation occuring in the electroweak phase transition when the scalar field is identified with the Higgs field. We calculate the determinant exactly and find an approximation (essentialy of the gradient expansion type) that reproduces the exact results very well if the fermion mass $m_{F}$ becomes larger than the inverse of the size $R$ of the background field configuration. The results are given for 2,3 and 4 Euclidean dimensions.
\end{abstract}

\footnotetext{
${ }^{1}$ e-mail baacke@het.physik.uni-dortmund.de

${ }^{2}$ On leave of absence from Dept. of Physics, Niigata University, Niigata, Japan

${ }^{3}$ e-mail_so@het.physik.uni-dortmund.de

${ }^{4}$ e-mail suerig@het.physik.uni-dortmund.de
} 


\section{Introduction}

The fermion determinant in an external Higgs field is a quantity that enters semiclassical rates [[1, 2] for sphaleron [3, 4, 5] and instanton [6] transitions as well as for the nucleation of bubbles or droplets [7, 8] in first order phase transitions, as possibly the electroweak phase transition occuring in the early universe. The classical action is usually minimized by spherically symmetric field configurations, the mass term produced by the Higgs field is then given by a radial profile $\phi(x)=$ $f(r)$. We will consider here field configurations that approach the broken Higgs phase as $r \rightarrow \infty$.

The exact calculation of such determinants has to be performed numerically since usually even the background field configuration is only given as a numerical profile. A very efficient method based on a theorem on functional determinants [9] has been developed recently by V. G. Kiselev and one of the authors (J.B.) [10]. We will apply this method here for evaluating the fermion determinant exactly in 2, 3 and 4 Euclidean dimensions.

It is certainly useful to dispose of analytical approximations to the fermion determinant, especially if the back reaction of this determinant on the dynamics of the Higgs field is being considered in the form of an effective action. It is our main purpose here to present such an approximation and, using our exact results, to demonstrate that it approximates the fermion determinant very well for sufficiently large fermion masses. This covers indeed the most relevant case since the effective action induced by fermion background fields behaves essentially as $m_{F}^{D}$ where $D$ is the Euclidean dimension and so is most relevant for heavy fermions.

The paper is organized as follows: In section 2 we recall some general relations and definitions. In section 3 we present the spherical decomposition of the relevant Dirac equation in $D=2,3$ and 4 and discuss the numerical evaluation of the determinant. In section 4 we develop our approximation. The numerical and approximate analytical results are presented and discussed in section 5. Details on the Dirac operators and the partial wave decomposition are given in the Appendix.

\section{Basic Relations}

The Dirac operator for a fermion with Yukawa coupling $g_{Y}$ to an external scalar field takes, in Euclidean space, the form

$$
\mathcal{D}=\gamma_{\mu} \partial_{\mu}-m_{F} \phi(x)=\mathcal{D}^{(0)}-m_{F}(\phi(x)-1)
$$

where we have normalized the "Higgs" field to its vacuum expectation value $v$ so that $m_{F}=g_{Y} v$ and where $\mathcal{D}^{(0)}$ is the free Dirac operator in the "broken 
Higgs phase" where $\phi(x)=1$. The gamma matrices are hermitean and satisfy $\gamma_{\mu} \gamma_{\nu}+\gamma_{\nu} \gamma_{\mu}=2 \delta_{\mu \nu}$. We also define the positive definite operator

$$
\mathcal{M}=\mathcal{D}^{\dagger} \mathcal{D}=-\partial^{2}+m_{F}^{2}+\mathcal{V}(x)
$$

where the potential or vertex matrix $\mathcal{V}$ takes the form

$$
\mathcal{V}(x)=m_{F}^{2}\left(\phi(x)^{2}-1\right)+m_{F} \gamma_{\mu} \partial_{\mu} \phi(x)
$$

We can evaluate the determinant of the Dirac operator, normalized to the free Dirac operator in the form

$$
\begin{gathered}
\ln \operatorname{det}\left(\mathcal{D} / \mathcal{D}^{(0)}\right)=\frac{1}{2} \ln \mathcal{J} \\
\mathcal{J}=\operatorname{det}\left(\frac{-\partial^{2}+m_{F}^{2}+V(x)}{-\partial^{2}+m_{F}^{2}}\right)
\end{gathered}
$$

Furthermore the fermionic fluctuation determinant is related to the corresponding one-loop effective action by the relation

$$
S_{\text {eff }}[\phi]=-\frac{1}{2} \ln \mathcal{J}
$$

The case $D=2$ could be relevant for lattice simulations of bubble nucleation at low temperature. The case $D=3$ will be considered with hindsight of an application as high temperature limit of the four dimensional theory. The four dimensional case which would be relevant to bubble formation at low temperatures (instanton type transition). The details on the $\gamma$ matrices are given in Appendix A.

\section{Numerical Computation of the Fluctuation Determinant}

For spherically symmetric background fields $\phi(x)=f(r)$ the determinant decomposes into contributions of partial waves in the form

$$
\ln \mathcal{J}=\sum d_{\lambda}(D) \ln J_{\lambda}
$$

where $\lambda$ denotes a coupled channel of partial waves, $J_{\lambda}$ is the partial wave determinant and $d_{\lambda}(D)$ is the degeneracy which depends on the dimension $D . J_{\lambda}$ is related to the partial wave reduction of the operator $\mathcal{M}$ which takes the general form

$$
\begin{aligned}
\mathbf{M}_{\lambda} & =\mathbf{M}_{\lambda}^{(0)}+\mathbf{V}(r) \\
\mathbf{M}_{\lambda}^{(0)} & =-\frac{d^{2}}{d r^{2}}-\frac{D-1}{r} \frac{d}{d r}+\frac{\mathbf{L}_{\lambda}^{2}}{r^{2}}+m_{F}^{2}
\end{aligned}
$$


via

$$
J_{\lambda}=\operatorname{det}\left(\frac{\mathbf{M}_{\lambda}}{\mathbf{M}_{\lambda}^{(0)}}\right)
$$

The degeneracies $d_{\lambda}$, and the matrices $\mathbf{L}_{\lambda}^{2}$ specifying the centrifugal barrier and $\mathbf{V}(\mathbf{r})$ are given in Appendix A.

For the numerical computation of the determinant $J_{\lambda}$ we use the method described in Ref [10], generalized to a coupled channel system. It is based on a theorem on functional determinants [9] which we recall below. The theorem has been used for a similar purpose for the case of massless fermions [11]. In the following we consider the contribution of one channel $\lambda$.

Let $\psi_{i \alpha}(r)$ be a fundamental system solutions of the differential equation

$$
\mathbf{M}_{\lambda} \psi_{i \alpha}=0
$$

and let $\psi_{i \alpha}^{(0)}$ be their free counterparts, both regular at $r=0$. Since there are as many independent solutions labelled by $\alpha$ as there are components labelled by $i$ these fundamental systems form quadratic matrices denoted by $\Psi=\left\{\psi_{i \alpha}\right\}$ and $\Psi^{(0)}=\left\{\psi_{i \alpha}^{(0)}\right\}$ respectively. Let furthermore the solutions be normalized such that

$$
\lim _{r \rightarrow 0} \Psi(r)\left(\Psi^{(0)}(r)\right)^{-1}=\mathbf{1} .
$$

Then according to the theorem on functional determinants [9] the partial wave determinant is obtained as

$$
\operatorname{det}\left(\frac{\mathbf{M}_{\lambda}}{\mathbf{M}_{\lambda}^{(0)}}\right)=\lim _{r \rightarrow \infty} \frac{\operatorname{det} \Psi(r)}{\operatorname{det} \Psi^{(0)}(r)}
$$

In the cases considered here - the Dirac operator with a scalar mass term in two, three and four dimensions - the partial wave reduction results in simple $(2 \times 2)$ systems. The "free" differential operator $\mathbf{M}_{\lambda}^{(0)}$ is diagonal and the solutions are modified Bessel functions which be denote as $b_{\lambda}$ for the upper component; their detailed form is given in Appendix A (see Eq. (A.16)). The solution for the lower component ist $b_{\lambda+1}$ in all cases considered here.

The solutions of the full operator $\mathbf{M}_{\lambda}$ are now written as

$$
\psi_{i \alpha}(r)=b_{\lambda+i-1}(r)\left(\delta_{i \alpha}+h_{i \alpha}(r)\right)
$$

where the boundary condition at $r=0$ requires the functions $h_{i \alpha}(r)$ to vanish there. Since the Bessel functions can be taken out of the rows of the matrix $\psi$ when forming the determinant they cancel in $J_{\lambda}$ and we have simply

$$
J_{\lambda}=\lim _{r \rightarrow \infty} \operatorname{det}(\mathbf{1}+\mathbf{h}(r)) .
$$

As in [10] we write directly a differential equation for the functions $h_{i \alpha}$. It takes the form

$$
\boldsymbol{\Delta}_{\lambda} \mathbf{h}=\mathbf{V}_{\lambda}(\mathbf{1}+\mathbf{h})
$$


where the differential operator $\boldsymbol{\Delta}_{\lambda}$ and the modified potential $\mathbf{V}_{\lambda}$ are defined in Eqs. (A.18-A.20). This differential equation is inhomogeneous and $\mathbf{h}$ may be expanded w.r.t. orders in the potential V. Denoting the contribution of order $k$ in the potential as $\mathbf{h}^{(k)}$ we introduce also the contributions summed from $k$ to $\infty$ as

$$
\mathbf{h}^{\overline{(k)}}=\sum_{l=k}^{\infty} \mathbf{h}^{(l)}
$$

so that $\mathbf{h}=\mathbf{h}^{\overline{(1)}}$. The differential equation can then be used to get the functions $\mathbf{h}$ of various orders recursively via

$$
\begin{aligned}
\boldsymbol{\Delta}_{n} \mathbf{h}^{(k+1)} & =\mathbf{V}_{n} \mathbf{h}^{(k)} \\
\boldsymbol{\Delta}_{n} \mathbf{h}^{\overline{(k+1)}} & =\mathbf{V}_{n} \mathbf{h}^{\overline{(k)}}
\end{aligned}
$$

where further $\mathbf{h}^{(0)} \equiv \mathbf{1}$. Using these contributions of specific order in $\mathbf{V}$ it is easy to get rid of the leading, divergent contributions to $\ln J_{\lambda}$ and therefore to $\ln \mathcal{J}$. This procedure has been discussed in [10] and can be performed analogously for a coupled system: In $D=2$ and $D=3$ the contribution of order $k=1$ has to be omitted, in $D=4$ the orders $k=1$ and 2 ; the finite parts of $\ln J_{\lambda}$ are then given by

$$
\left(\ln J_{\lambda}\right)^{\overline{(2)}}=\lim _{r \rightarrow \infty}\left(\operatorname{Tr}[\ln (\mathbf{1}+\mathbf{h})-\mathbf{h}]+\operatorname{Tr}\left(\mathbf{h}^{\overline{(2)}}\right)\right)
$$

and

$$
\begin{aligned}
\left(\ln J_{\lambda}\right)^{\overline{(3)}} & =\lim _{r \rightarrow \infty}\left(\operatorname{Tr}\left[\ln (\mathbf{1}+\mathbf{h})-\mathbf{h}+\frac{1}{2} \mathbf{h}^{2}\right]\right. \\
& \left.+\operatorname{Tr}\left(\mathbf{h}^{\overline{(3)}}\right)-\frac{1}{2} \operatorname{Tr}\left(\mathbf{h}^{\overline{(2)}}\left(\mathbf{h}^{\overline{(1)}}+\mathbf{h}^{(1)}\right)\right)\right)
\end{aligned}
$$

The matrices in square brackets can be diagonalized in terms of the eigenvalues of $\mathbf{h}$ and reduce then to "subtracted logarithms" which can be evaluated with arbitrary precision. The remaining terms are separately of order $\overline{(2)}$ and of $\operatorname{order} \overline{(3)}$ in the first and second equation respectively. These expressions do not contain therefore any dangereous small differences of large numbers. The total fluctuation determinants are denoted correspondingly as $(\ln \mathcal{J})^{\overline{(2)}}$ and $(\ln \mathcal{J})^{\overline{(3)}}$.

The divergent contributions have to be regularized and renormalized and this depends on the specific model under consideration. These contributions can easily be calculated analytically, at most one needs the Fourier transform of the profile (see e.g. [10 where this step was included). Here we are interested in the comparison with the large $m_{F}$ expansion and for this purpose it is sufficient to consider those finite parts of the fluctuation determinant which are obtained by omitting the renormalization parts completely.

The numerical computation has been carried out with all the technical details as described in Ref. [10]. The results will be discussed in section 5 . 


\section{The Large Mass Approximation}

If the fermion mass $m_{F}$ becomes much larger than the inverse size $R^{-1}$ of the background field configuration one expects the Dirac operator to "seize" the background field only locally. Therefore it should be possible to expand the fluctuation determinant with respect to derivatives which on dimensional grounds results in an expansion with respect to $1 / m_{F}$. Various techniques are available. While the heat kernel expansion is used frequently we use the ordinary Feynman graph or resolvent expansion here. While heat kernel expansions are often broken off at a fixed power of the heat kernel time we will keep track here of all terms of the leading and next-to-leading power of the mass $m_{F}$.

The fermion determinant can be written exactly as

$$
\ln \mathcal{J}=\operatorname{Tr} \ln \left(\mathbf{1}+\mathbf{G}^{(0)} \mathcal{V}\right)
$$

where the free Green function is defined as

$$
\mathcal{M G}^{(0)}=1
$$

We define the Fourier transform of the potential $\mathcal{V}$ as

$$
\widetilde{\mathcal{V}}(\mathbf{q})=\int d^{D} x \mathcal{V}(\mathbf{x}) \exp (-i \mathbf{q} \mathbf{x})
$$

The fluctuation determinant can then be expanded as

$$
\ln \mathcal{J}=\sum_{n=1}^{\infty} \operatorname{Tr} \frac{(-1)^{n+1}}{n} \int \frac{d^{D} p}{(2 \pi)^{D}} \prod_{j=1}^{n} \int \frac{d^{D} q_{j}}{(2 \pi)^{D}} \frac{\tilde{\mathcal{V}}\left(\mathbf{q}_{j}\right)}{\left(\mathbf{p}+\mathbf{Q}_{j}\right)^{2}+m_{F}^{2}}(2 \pi)^{D} \delta^{D}\left(\mathbf{Q}_{n}\right)
$$

where

$$
\mathbf{Q}_{j}=\sum_{l=1}^{j} \mathbf{q}_{l}
$$

The range of the integration in the variables $\mathbf{q}_{j}$ is determined by the inverse range of the external field configuration $R^{-1}$. If $m_{F} \gg R^{-1}$ the scale for the integration in $\mathbf{p}$ is set by the fermion mass $m_{F}$. This integration yields therefore a factor $m_{F}^{(D-2 n)}$. Recalling the form of the potential

$$
\mathcal{V}=m_{F}^{2}\left(\phi^{2}-1\right)+m_{F} \gamma \partial \phi
$$

we see that in leading order the product over the potentials yields a factor $m_{F}^{2 n}$ so that the leading behaviour of $\ln \mathcal{J}$ is $m_{F}^{D}$ ?. The next-to-leading order term is obtained by taking into account the first nonleading terms in the expansion

\footnotetext{
${ }^{1}$ Note that this power counting is different in the case of chiral mass terms of the form $m_{0} \exp \left(i \gamma_{5} \phi_{a} \tau_{a}\right)$ where only the gradient term linear in $m_{0}$ appears in $\mathcal{V}$.
} 
of the denominators and of the trace of the product over the potentials. Using symmetric integration we have

$$
\begin{aligned}
\prod_{j=1}^{n} \frac{1}{\left(\mathbf{p}+\mathbf{Q}_{j}\right)^{2}+m_{F}^{2}} & \simeq \frac{1}{\left(\mathbf{p}^{2}+m_{F}^{2}\right)^{n}}\left[1-\sum_{l=1}^{n} \frac{\mathbf{Q}_{l}^{2}}{\left(\mathbf{p}^{2}+m_{F}^{2}\right)}\right. \\
& \left.+\frac{4}{D} \frac{\mathbf{p}^{2}}{\left(\mathbf{p}^{2}+m_{F}^{2}\right)^{2}}\left(\sum_{l>l^{\prime}} \mathbf{Q}_{l} \mathbf{Q}_{l^{\prime}}+\sum_{l=1}^{n} \mathbf{Q}_{l}^{2}\right)\right]
\end{aligned}
$$

up to terms of order $m_{F}^{(-2 n-4)}$. Similarly we have

$$
\begin{aligned}
\operatorname{Tr} \prod_{j} \tilde{\mathcal{V}}\left(\mathbf{q}_{j}\right) & \simeq \nu_{F} m_{F}^{2 n}\left[\prod_{j}\left(\phi^{2-1}-1\right)\left(\mathbf{q}_{j}\right)\right. \\
& \left.-\frac{1}{m_{F}^{2}} \sum_{l>l^{\prime}} \mathbf{q}_{l} \mathbf{q}_{l^{\prime}} \phi\left(\mathbf{q}_{l}\right) \phi\left(\mathbf{q}_{l^{\prime}}\right) \prod_{j \neq l, l^{\prime}}\left(\widetilde{\phi^{2}-1}\right)\left(\mathbf{q}_{j}\right)\right]
\end{aligned}
$$

up to terms of order $m_{F}^{(2 n-4)}$. Here $\nu_{F}$ is 2 for $D=2$ and 4 in $D=3$ and $D=4$. Inserting these expansions one obtains after transforming back to $x$ space

$$
\begin{aligned}
\ln \mathcal{J} & =\nu_{F} m_{F}^{D} \sum_{n=1}^{\infty} \frac{(-1)^{n+1}}{n} \int d^{D} x\left\{\left(I(D, 0, n)\left(\phi^{2}-1\right)^{n}\right.\right. \\
& +\frac{n(n-1)}{2 m_{F}^{2}} I(D, 0, n)\left(\phi^{2}-1\right)^{n-2}\left(\partial_{\mu} \phi\right)^{2} \\
& -\frac{2(n+1) n(n-1)}{3 m_{F}^{2}} I(D, 0, n+1) \phi^{2}\left(\phi^{2}-1\right)^{n-2}\left(\partial_{\mu} \phi\right)^{2} \\
& \left.+\frac{2(n+2)(n+1) n(n-1)}{3 D m_{F}^{2}} I(D, 1, n+2) \phi^{2}\left(\phi^{2}-1\right)^{n-2}\left(\partial_{\mu} \phi\right)^{2}\right\}
\end{aligned}
$$

where

$$
I(D, m, n)=\int \frac{d^{D} l}{(2 \pi)^{D}} \frac{\left(\mathbf{l}^{2}\right)^{m}}{\left(\mathbf{l}^{2}+1\right)^{n}}
$$

The sum over $n$ can be performed after evaluating explicitly the (dimensionless) integrals $I(D, m, n)$ in the various dimensions. The first few terms are however formal only: the integrals are divergent. As for the exact computation we have to omit, for $D=2$ and $D=3$ the order $n=1$ and for $D=4$ the orders $n=1$ and 2 . We denote the summation over $n=n_{0}, . . \infty$ by the symbol $\overline{\left(n_{0}\right)}$ as already in the previous section.

Writing the result in the form of an effective action we have for $D=2$

$$
\begin{aligned}
S_{\text {eff }}^{\overline{(2)}}[\phi]=-\frac{1}{2} \ln \mathcal{J}^{\overline{(2)}} \simeq & \frac{m_{F}^{2}}{4 \pi} \int d^{2} x\left(1+\phi^{2}\left(\ln \left(\phi^{2}\right)-1\right)\right) \\
& +\frac{1}{24 \pi} \int d^{2} x \frac{\left(\partial_{\mu} \phi\right)^{2}}{\phi^{2}}
\end{aligned}
$$


for $D=3$

$$
\begin{aligned}
S_{\text {eff }}^{\overline{(2)}}[\phi]=-\frac{1}{2} \ln \mathcal{J}^{\overline{(2)}} \simeq & \frac{m_{F}^{3}}{3 \pi} \int d^{3} x\left(|\phi|^{3}-\frac{3}{2}\left(\phi^{2}-1\right)-1\right) \\
& +\frac{m_{F}}{12 \pi} \int d^{3} x \frac{\left(\partial_{\mu} \phi\right)^{2}}{|\phi|}
\end{aligned}
$$

and for $D=4$

$$
\begin{aligned}
S_{\text {eff }}^{\overline{(3)}}[\phi]=-\frac{1}{2} \ln \mathcal{J}^{\overline{(3)}} \simeq & -\frac{m_{F}^{4}}{16 \pi^{2}} \int d^{4} x\left(\phi^{4} \ln \left(\phi^{2}\right)-\frac{3}{2}\left(\phi^{2}-1\right)^{2}-\left(\phi^{2}-1\right)\right) \\
& -\frac{m_{F}^{2}}{16 \pi^{2}} \int d^{4} x\left(\partial_{\mu} \phi\right)^{2}\left(\ln \left(\phi^{2}\right)-\frac{2}{3}\left(\phi^{2}-1\right)\right)
\end{aligned}
$$

\section{Results and Conclusions}

We have evaluated the exact fluctuation determinants described in the previous sections in $D=2,3$ and 4 for two different typical profiles, a "small" and a "large" bubble. For convenience we used an analytical expression for the radial profile

$$
f(r)=\left(1+\exp \left(-\sqrt{r^{2}+1}+\sqrt{R^{2}+1}\right)\right)^{-1}
$$

This function behaves near $r=0$ as $c_{1}+c_{2} r^{2}$ in analogy to the solutions of the typical field equations for the Higgs field, for $r \rightarrow \infty$ it behaves as $1-c_{3} \exp (-r)$ as it would behave for a Higgs field of mass 1 . $R$ determines the point $f(r)=1 / 2$ and therefore the typical size of the profile. We have chosen $R=2$ and $R=6$. In the second case the function $f(r)$ goes to .006 at $r=0$ so that the symmetric Higgs phase is almost reached. This is a situation where breaking off the expansion in $\left(\phi^{2}-1\right)$ at a finite power of this quantity would lead to a poor approximation. The profiles are displayed in Fig. 1.

The results of the exact and approximate computations are presented in Figs. $2-7$, both for the small and large sized profiles and for $\mathrm{D}=2,3$ and 4 .

The precision of the exact computation can be estimated from the convergence of the partial wave expansion, from varying the grid density for the radial variable (we have typically 1000 points in the range $0<r<15$ ) etc. to be better than of $10^{-3}$. The agreement with the estimate at large fermion masses further corroborates this estimate. The precision becomes poorer if $m_{F} R$ exceeds a value of $\sim 12$ since then the asymptotic regime of the partial wave series, requiring $l \gg m_{F} R$ is not yet reached at our maximal value of $l=25$; while the higher partial waves are included using a fit $a / l^{3}+b / l^{4}$ the parameters of this fit are then not reliable.

In the case $D=2$ and $R=6$ a bound state eigenvalue approaches 0 in the $\mathrm{S}$-wave and the calculation becomes numerically delicate at $m_{F}>3$. The poor 
agreement in next-to-leading order between asymptotic estimate and exact results which is apparent in Fig. 3 is however not due to the numerics which we have checked in all aspects. The $\phi^{-2}$ singularity in the kinetic term of the effective action for $D=2$, Eq. (4.11), indicates a bad convergence of the $1 / m_{F}$ expansion if $\phi$ becomes small, even if, as on the present case, the integral exists. In all other cases, for both profiles and all dimensions, the first two terms of the $1 / m_{F}$ expansion agree very well with the exact results for sufficiently large $m_{F}$. The asymptotic regime sets in earliest for $D=4$.

In conclusion we have found in general an excellent agreement between our exact and approximate evaluations of the fluctuation determinant of a heavy fermion coupled to an external Higgs field, the singularities of the gradient terms at $\phi(x)=0$ are however potentially dangereous and need further consideration. The comparison corroborates the precision of the numerical method. The analytic approximation has the form of an effective action including all terms with at most two derivatives in the external field. Its should be interesting to include it into the action from which the classical profiles are determined.

\section{Acknowledgements}

We have pleasure in thanking Valeri Kiselev for many useful discussions. Hiroto So thanks the Japanese Ministry of Education for a grant. Jürgen Baacke thanks the DESY, where part of this work was performed, for hospitality.

\section{A Appendix}

We shall summarize the notations and formulas in $\mathrm{D}=2,3$ and 4 . The Euclidean Dirac operator, $\mathcal{D}$, is defined as

$$
\mathcal{D}=\gamma_{\mu} \partial_{\mu}-m_{F} \phi(x)
$$

As gamma matrix convention for $D=2$ we take

$$
\begin{aligned}
\gamma_{1} & =\sigma_{1} \\
\gamma_{2} & =\sigma_{2} \\
\gamma_{5} & =-\sigma_{3} \\
\gamma_{\mu} \gamma_{\nu} & =\delta_{\mu \nu}-i \epsilon_{\mu \nu} \gamma_{5} .
\end{aligned}
$$

For $D=3$ f and $D=4$ we use

$$
\gamma_{\mu}=\left(\begin{array}{cc}
0 & \Sigma_{\mu} \\
\Sigma_{\mu}^{\dagger} & 0
\end{array}\right)
$$

\footnotetext{
${ }^{2}$ The three dimensional theory is considered here as a three dimensional reduction of a four dimensional one as it occurs in finite temperature quantum field theory
} 
with

$$
\Sigma_{\mu}=\left\{\begin{array}{cl}
1_{2 \times 2} & \mu=4 \\
i \sigma_{a} & \mu=a=1 \sim 3
\end{array}\right.
$$

$\gamma_{5}$ is then

$$
\gamma_{5}=\left(\begin{array}{cc}
-1_{2 \times 2} & 0 \\
0 & 1_{2 \times 2}
\end{array}\right)
$$

This is just the chiral representation. For a field configuration $\phi$ which depends only on the radius, $\phi(x)=f(r)$ the "squared" Dirac operator $\mathcal{M}$ defined in Eq. (2.2) is given by

$$
\begin{aligned}
\mathcal{M} & =-\partial^{2}+m_{F}^{2}+m_{F}^{2}\left(f(r)^{2}-1\right)+m_{F} f^{\prime}(r) \hat{x}_{\mu} \gamma_{\mu} \\
& =-\frac{\partial^{2}}{\partial r^{2}}-\frac{D-1}{r} \frac{\partial}{\partial r}+\frac{L^{2}}{r^{2}}+m_{F}^{2}+\mathcal{V}(r)
\end{aligned}
$$

where $\hat{x}_{\mu}$ is the unit vector of the coordinate $x_{\mu}$, potential $\mathcal{V}$ becomes

$$
\mathcal{V}=m_{F}^{2}\left(f^{2}-1\right)+m_{F} f^{\prime} \hat{x}_{\mu} \gamma_{\mu}
$$

and $L^{2} / r^{2}$ is the centrifugal potential.

We take as a basis for the solutions of the Dirac equation in $D=2$ the spinors

$$
\Psi=\left(\begin{array}{c}
\psi_{L} \\
\psi_{R}
\end{array}\right)=\left(\begin{array}{c}
u(r) e^{i n \varphi} \\
v(r) e^{i(n+1) \varphi}
\end{array}\right)
$$

where the angular momentum $n$ takes the values $n=0, \pm 1, \pm 2 \ldots$ For $D=3$ we use the spinors

$$
\Psi=\left(\begin{array}{c}
\psi_{L} \\
\psi_{R}
\end{array}\right)=\left(\begin{array}{c}
u(r) \chi \\
i v(r) \hat{x}_{a} \sigma_{a} \chi
\end{array}\right) .
$$

For the four dimensional theory we take

$$
\Psi=\left(\begin{array}{c}
\psi_{L} \\
\psi_{R}
\end{array}\right)=\left(\begin{array}{c}
u(r) \chi \\
v(r) \hat{x}_{\mu} \Sigma_{\mu}^{\dagger} \chi
\end{array}\right)
$$

$\chi$ is a 2-component spinor multiplied by a spherical harmonic function and

$$
L^{2} \chi=4 j(j+1) \chi
$$

where $j(=0,1 / 2,1 \ldots)$ is the magnitude of 'angular momentum' in $\mathrm{D}=4$ and

$$
L^{2} \chi=j(j+1) \chi
$$

where $j(=0,1, .$.$) is the magnitude of angular momentum in \mathrm{D}=3$. Then the 'squared' Dirac equations are

$$
-u^{\prime \prime}-\frac{1-2 \beta}{r} u^{\prime}+\frac{\lambda^{2}-\beta^{2}}{r^{2}} u+m_{F}^{2} u+m_{F}^{2}\left(f^{2}-1\right) u+m_{F} f^{\prime} v=0
$$




$$
-v^{\prime \prime}-\frac{1-2 \beta}{r} v^{\prime}+\frac{(\lambda+1)^{2}-\beta^{2}}{r^{2}} v+m_{F}^{2} v+m_{F}^{2}\left(f^{2}-1\right) v+m_{F} f^{\prime} u=0
$$

where

$$
\lambda= \begin{cases}|n|=0,1,2, . ., & \text { for } \mathrm{D}=2 \\ j+1 / 2=1 / 2,3 / 2,5 / 2, . ., & \text { for } \mathrm{D}=3 \\ 2 j=0,1,2, . ., & \text { for } \mathrm{D}=4\end{cases}
$$

and $\beta$ is $\frac{2-D}{2}$. In this basis the potential is simply the $(2 \times 2)$ matrix

$$
\mathbf{V}=\left(\begin{array}{cc}
m_{F}^{2}\left(f^{2}-1\right) & m_{F} f^{\prime} \\
m_{F} f^{\prime} & m_{F}^{2}\left(f^{2}-1\right)
\end{array}\right)
$$

For the free equation $(f=1)$, the regular solutions are

$$
\begin{aligned}
& \Psi_{1}=\left(\begin{array}{c}
b_{\lambda}\left(m_{F} r\right) \\
0
\end{array}\right) \\
& \Psi_{2}=\left(\begin{array}{c}
0 \\
b_{\lambda+1}\left(m_{F} r\right)
\end{array}\right)
\end{aligned}
$$

where

$$
b_{\lambda}\left(m_{F} r\right)=r^{\beta} I_{\lambda}\left(m_{F} r\right)
$$

and $I_{\lambda}\left(m_{F} r\right)$ is the modified Bessel function of order $\lambda$. Therefore, the solutions of the 'squared' Dirac equation are expressed as

$$
\psi_{i \alpha}=\left(\delta_{i \alpha}+h_{i \alpha}(r)\right) b_{n-1+i}\left(m_{F} r\right)
$$

where $\alpha$ labels the different solutions and $i$ the components of the solution. The functions $h_{i \alpha}(r)$, are determined by

$$
\Delta_{\lambda, i j} h_{j \alpha}=V_{\lambda, i j}\left(\delta_{j \alpha}+h_{j \alpha}\right)
$$

where

$$
\Delta_{\lambda, i j}=\left[\frac{d^{2}}{d r^{2}}+\left(\frac{D-1}{r}+2 m_{F} \frac{b_{\lambda-1+i}^{\prime}\left(m_{F} r\right)}{b_{\lambda-1+i}\left(m_{F} r\right)}\right) \frac{d}{d r}\right] \delta_{i j}
$$

and

$$
V_{\lambda, i j}=V_{i j} \frac{b_{\lambda-1+j}\left(m_{F} r\right)}{b_{\lambda-1+i}\left(m_{F} r\right)}
$$

The degeneracies of the solutions are given by

$$
d_{\lambda}(\mathrm{D})= \begin{cases}2, & \text { for } \mathrm{D}=2 \\ 2(2 \lambda+1), & \text { for } \mathrm{D}=3 \\ 2(\lambda+1)(\lambda+2), & \text { for } \mathrm{D}=4\end{cases}
$$




\section{References}

[1] J. S. Langer, Ann.Phys. (N.Y.) 41 (1967) 108; ibid. 54 (1969) 258

[2] I. Affleck, Phys. Rev. Let. 46 (1981) 388

[3] N. S. Manton, Phys. Rev. D28 (1983) 2019

[4] F. R. Klinkhamer and N. S. Manton, Phys. Rev. D30 (1984) 2212

[5] P. Arnold and L. McLerran, Phys. Rev. D36 (1987) 581; ibid. D37 (1988) 1020

[6] G. 't Hooft, Phys. Rev. D14 (1976) 3432

[7] S. Coleman, Phys. Rev. D15 (1977) 2929

[8] C. G. Callan and S. Coleman, Phys. Rev. D16 (1977) 1762

[9] S. Coleman, 'The Uses of Instantons' in "The Whys of Subnuclear Physics", A. Zichichi ed., Plenum Press, New York 1979; R. F. Dashen, B. Hasslacher, and A. Neveu, Phys.Rev. D10 (1974) 4114; I. M. Gel'fand and A. M. Yaglom, J. Math. Phys. 1 (1960) 48; R. H. Cameron and W. T. Martin, Bull. Am. Math. Soc. 51 (1945) 73; J. H. van Vleck, Proc. Nat. Acad. Sci. 14 (1928) 178

[10] J. Baacke and V. G. Kiselev, Phys. Rev. D48 (1993) 5648

[11] J. Avan and H. J. de Vega, Nucl. Phys. B269 (1986) 621; 


\section{Figure Captions}

Fig. 1 The Higgs field profiles. We plot the radial profiles given in Eq. (5.1) for $R=2$ and $R=6$ as used in our computations.

Fig. 2 The fermionic effective action $S_{e f f}^{\overline{(2)}} / m_{F}^{2}$ for $D=2, R=2$. The solid line represents the analytic approximation of Eq. (4.11), the numerical exact results are plotted as points $(\bullet)$ connected by straight dotted lines. The straight dashed line indicates the limit $m_{F} \rightarrow \infty$.

Fig. 3 The same as Fig. 2 for $R=6$.

Fig. 4 The fermionic effective action $S_{e f f}^{\overline{(2)}} / m_{F}^{3}$ for $D=3, R=2$. Description as for Fig. 2, the analytic approximation being given in Eq. (4.12).

Fig. 5 The same as Fig. 4 for $R=6$.

Fig. 6 The fermionic effective action $S_{e f f}^{\overline{(3)}} / m_{F}^{4}$ for $D=4, R=2$. Description as for Fig. 2, the analytic approximation being given in Eq. 4.13).

Fig. 7 The same as Fig. 6 for $R=6$. 


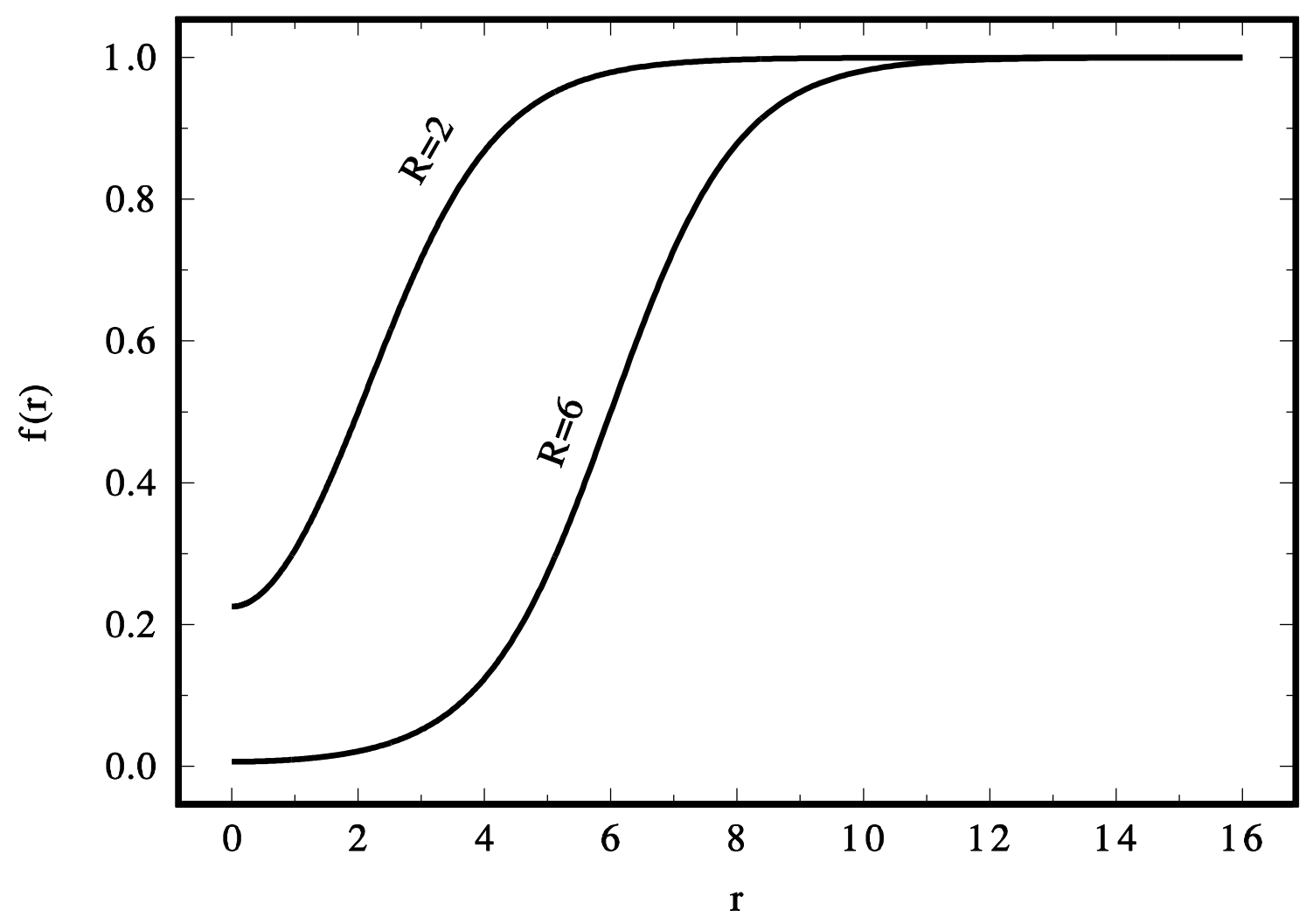

Figure 1 


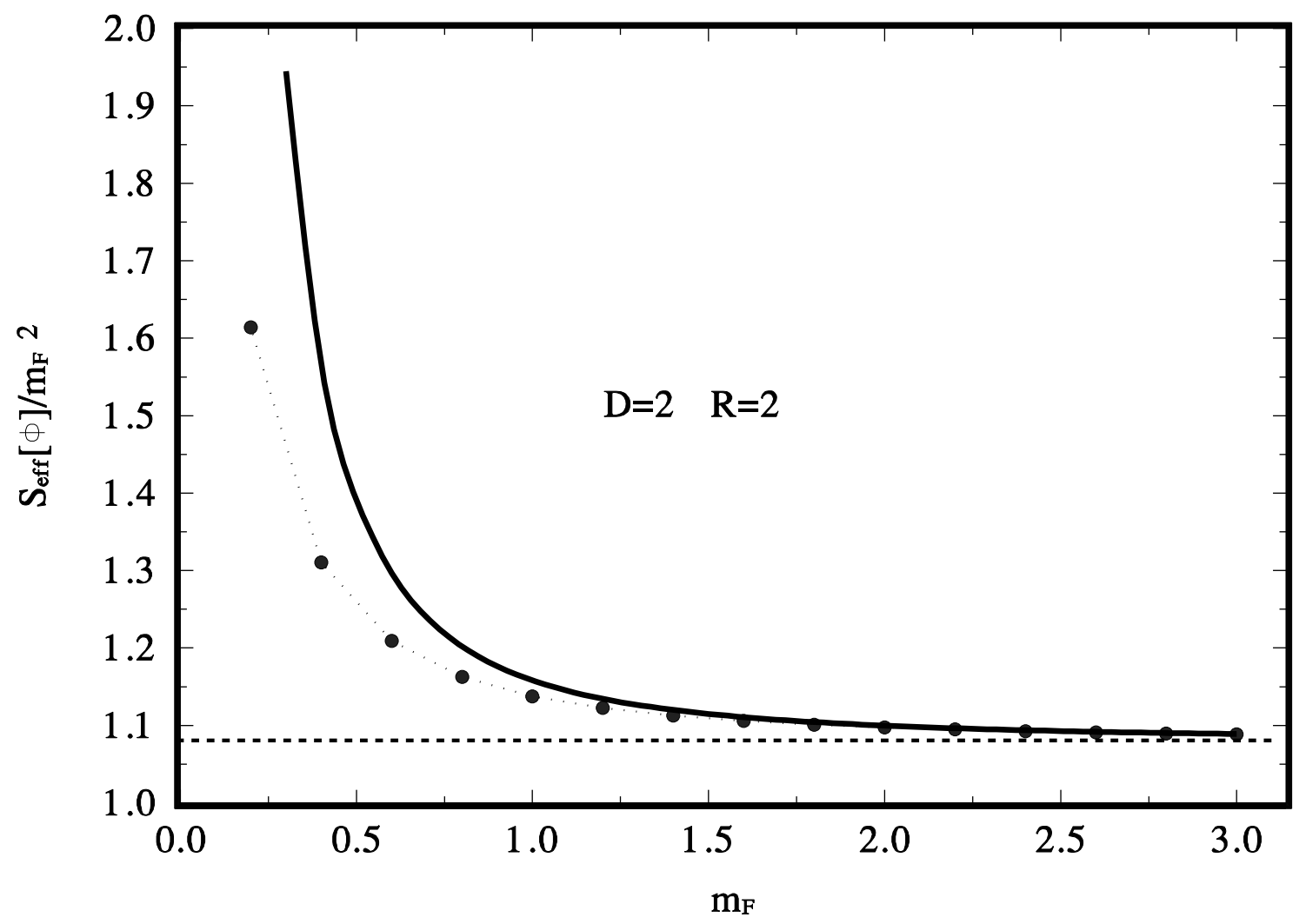

Figure 2 


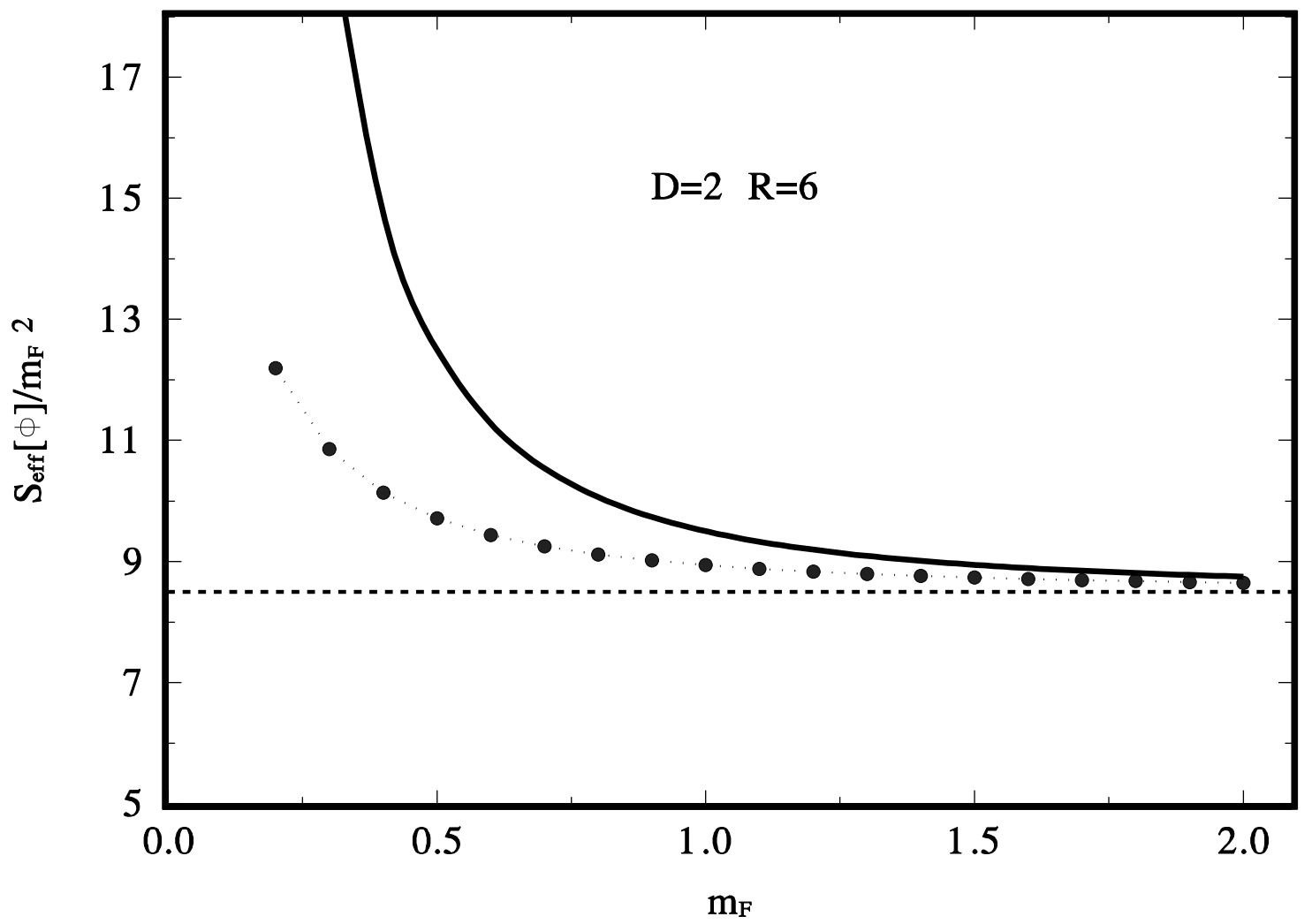

Figure 3 


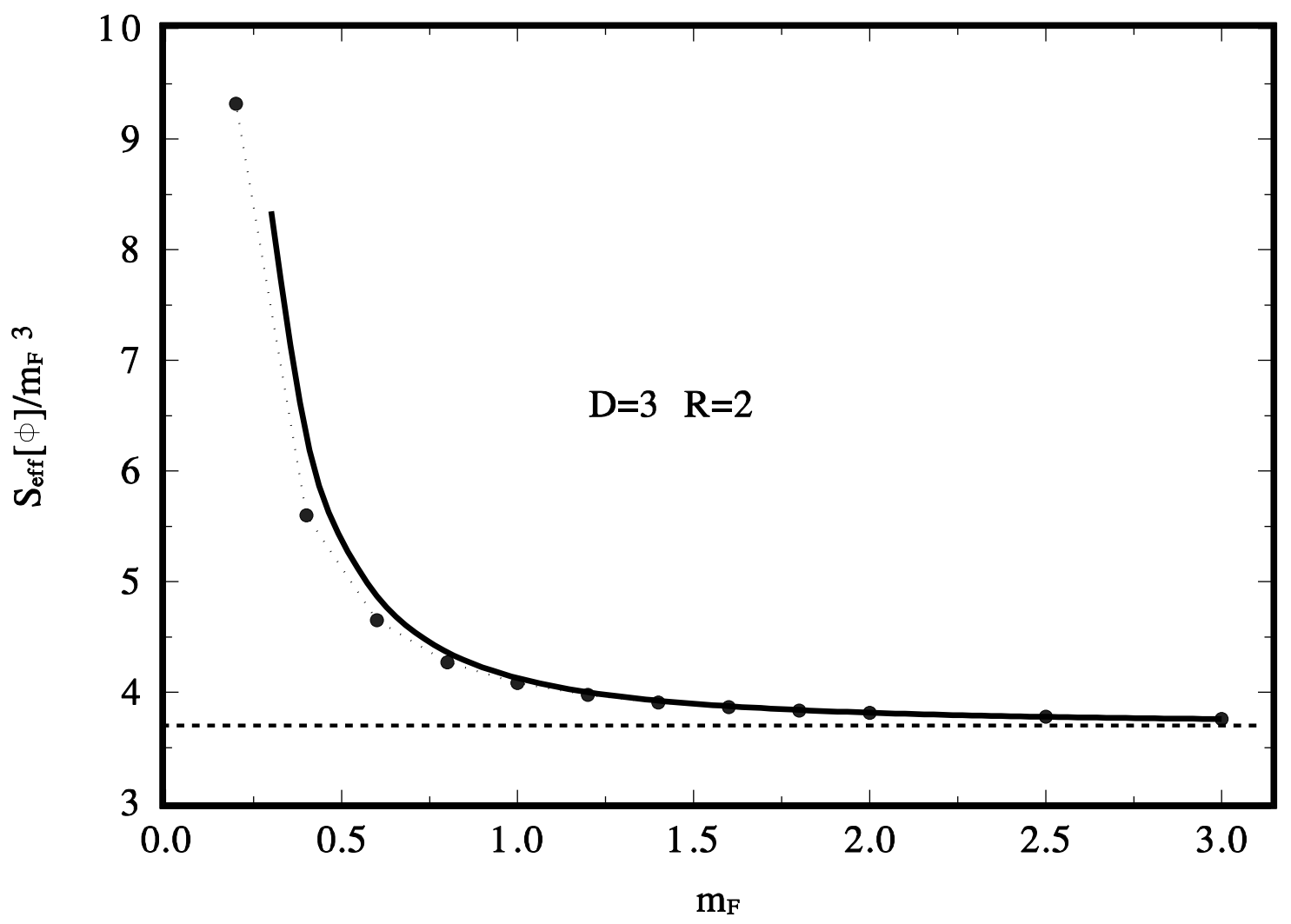

Figure 4 


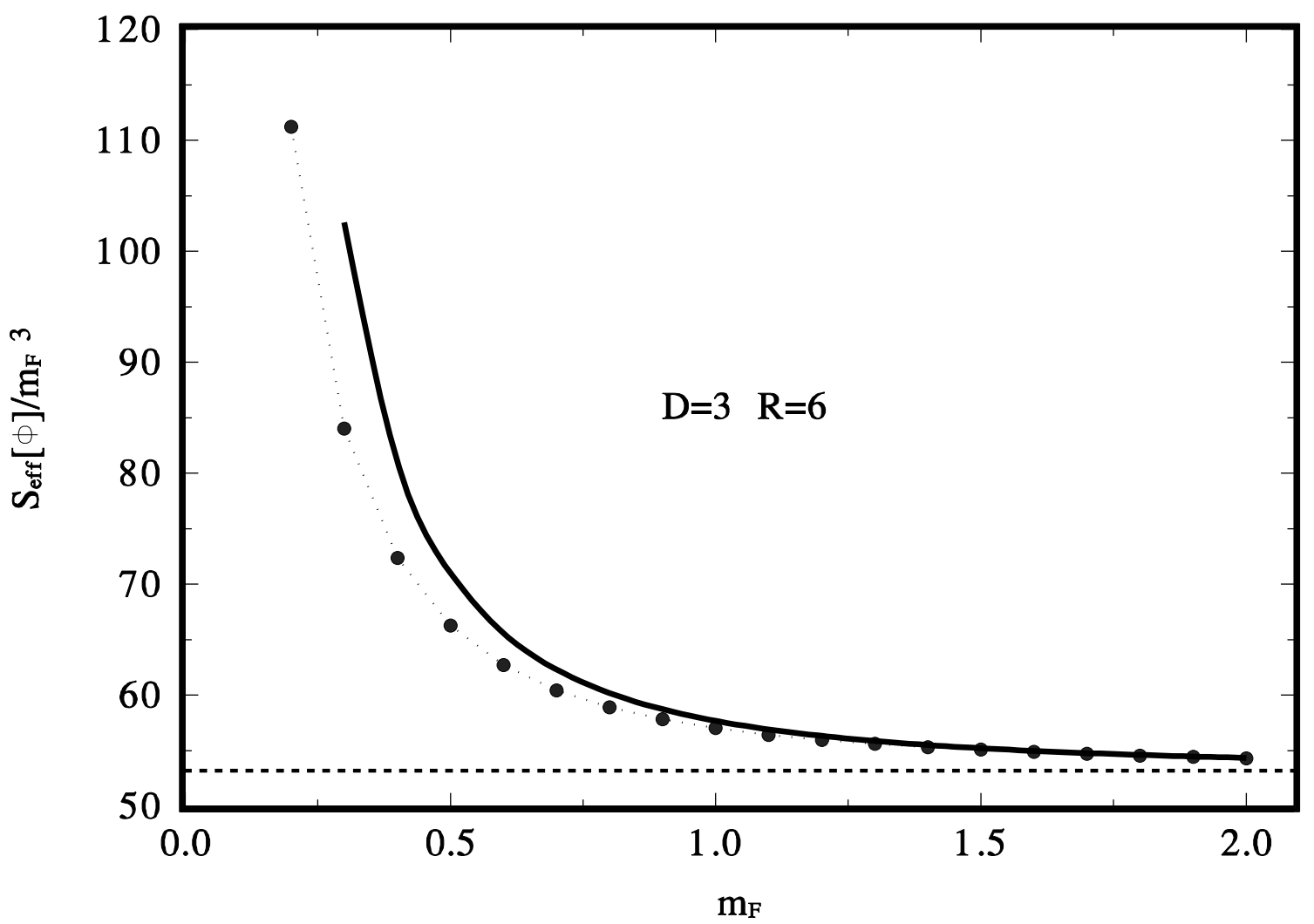

Figure 5 


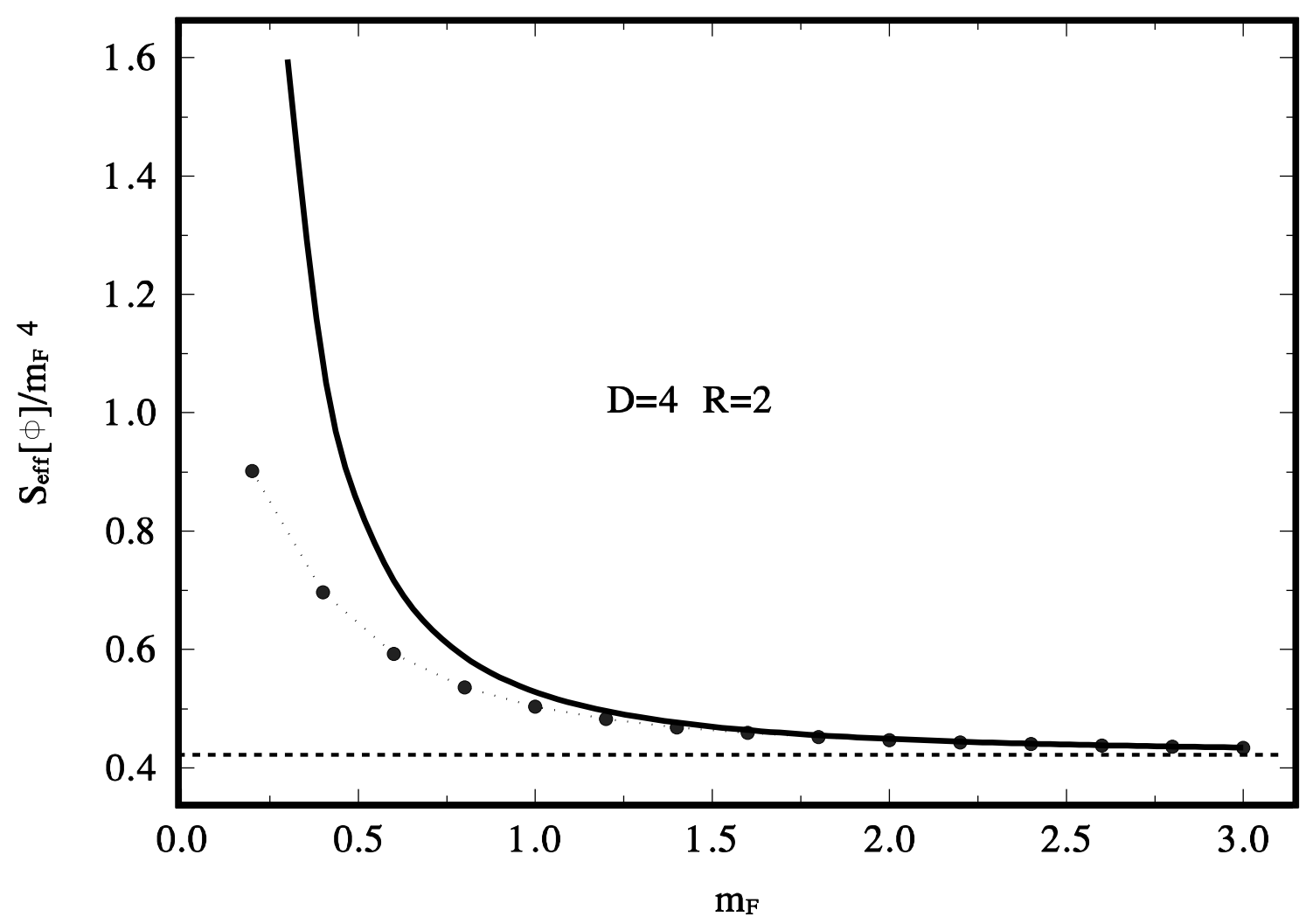

Figure 6 


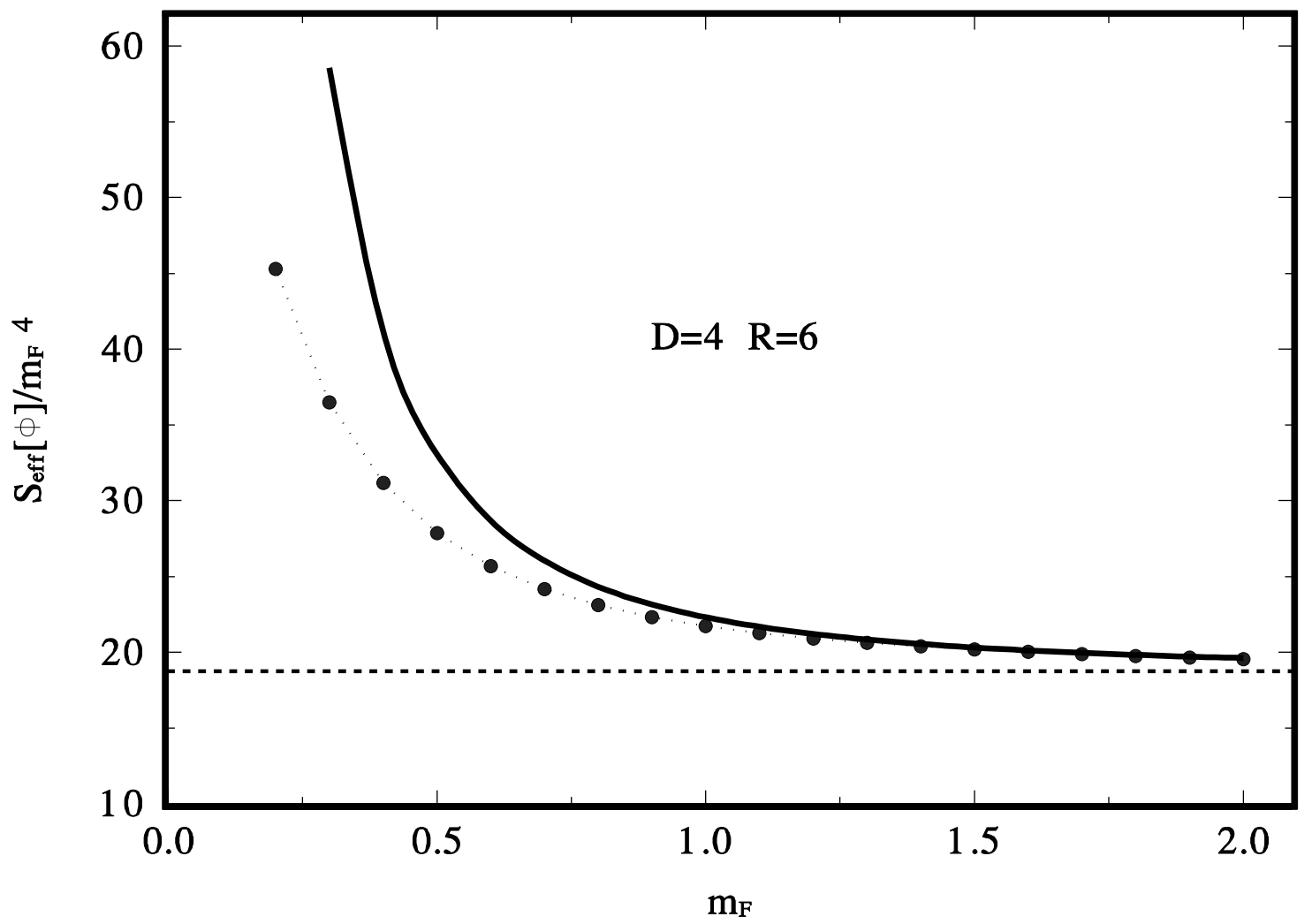

Figure 7 